\title{
A Proposed Non Linear Block Based Approach for Image De-Noising Using Wavelet
}

\author{
${ }^{1}$ Rajeev Bedi, ${ }^{2}$ Tajinder Singh ${ }^{3}$ S.K Gupta \\ ${ }_{1,2,3}$ BCET Gurdapur (Punjab)
}

\begin{abstract}
Coding of images or processing of images has played a momentous role in the success of digital communications and multimedia. Coding plays a major role is 3D mobile generation. The image processing methods have been exploited through the years to improve the quality of digital images. In the image processing system De-noising based coding is used extensively. As we know that, in low bit rate applications, the blocking artifacts problem arises, which severely reduce the visual quality of the image. Reducing blocking artifacts is essential to render the compressed visual data acceptable to the viewer. So, to detect and reduce noise from the image is a major task. So removing the noise from original signal is still a challenging problem for researchers. There have been several published algorithms and each approach has its assumptions, advantages, and limitations. For real-time applications like television, photo-phone, etc. it is essential to reduce the noise power as much as possible and to retain the fine details and the edges in the image as well. Moreover, it is very important to have very low computational complexity so that the filtering operation is performed in a short time for online and real-time applications. The main focus of this paper is first to define an algorithm and based on that a non linear threshold filter is described which not only preserve the actual image structures in the presence of different types of noises (Salt \& peepers, Speckle and Gaussian Noise) as well as it provide better PSNR, MSE, MAE, \& Time Complexity than others Classical algorithms.
\end{abstract}

Key words: De-noise, Wavelet, Threshold, Filter, Pixel.

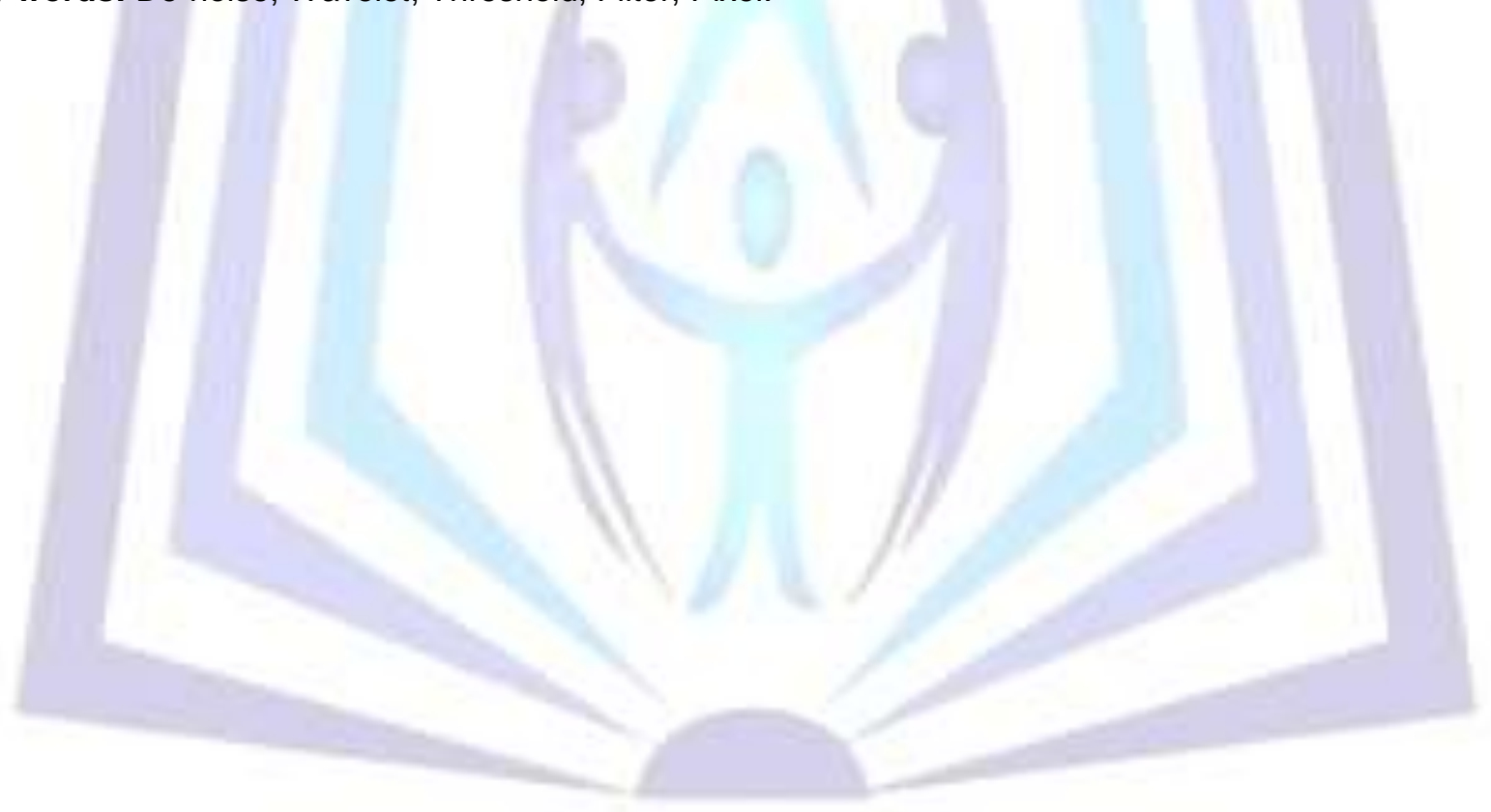

\section{Council for Innovative Research}

Peer Review Research Publishing System

Journal: INTERNATIONAL JOURNAL OF COMPUTERS \& TECHNOLOGY

Vol 5, No 3 


\section{INTRODUCTION}

In the digital image processing, an Image may be defined as a two-dimensional function as, $f(x, y)$, where $x$ and $y$ are spatial (plane) coordinates, and the amplitude of $f$ at any pair coordinates $(x, y)$ is called the intensity or gray level of the image at that point [2] [4]. When ( $x, y)$ and the amplitude values of $f$ are all finite, discrete quantities, we call the image a digital image as shown in figure 1. Therefore, a digital image is a two-dimensional array of small square regions known as pixels.

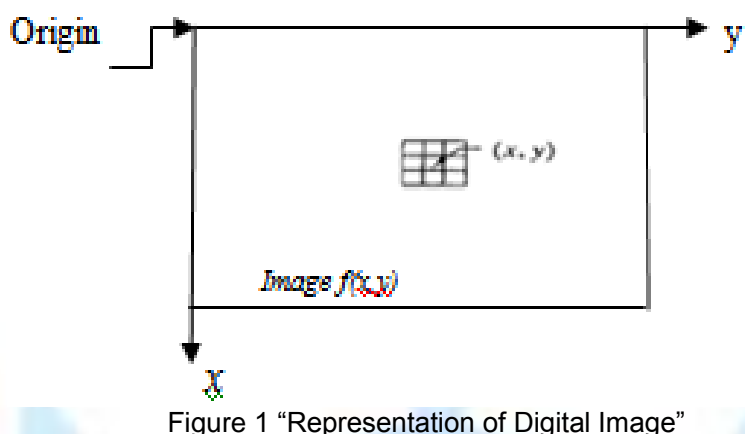

\section{CLASSIFICATION OF IMAGE DE-NOISING ALGORITHM}

Classification of an image de-noising algorithm can be done on the basis of spatial filtering scheme.

\section{Spatial Filtering:}

Noise from the digital images is removed by using spatial filters. These can be divided in two parts which are describes in the figure i.e.

Linear Filters

Non-Linear Filters

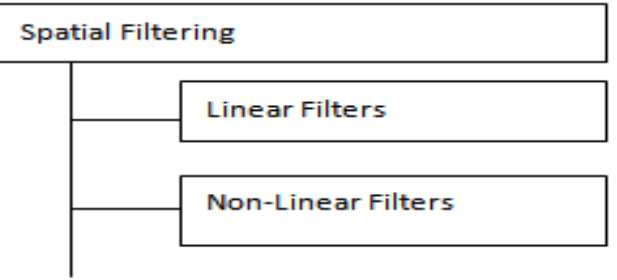

Figure 2 "Classification of Image De-Noising Algorithms"

\section{Non-Linear Filters}

With non-linear filters, the noise is removed without any attempts to explicitly identify it. Spatial filters employ a low pass filtering on groups of pixels with the assumption that the noise occupies the higher region of frequency spectrum. Generally spatial filters remove noise to a reasonable extent. In recent years, a variety of nonlinear median type filters such as weighted median [8], rank conditioned rank selection (RCRS) [9], and relaxed median (RM) [10] have been developed to remove the noise from digital images.

\section{Linear Filters}

A mean filter is the optimal linear filter for Gaussian noise in the sense of mean square error. Linear filters too tend to blur sharp edges, destroy lines and other fine image details, and Perform poorly in the presence of signal-dependent noise. The wiener filtering [11] method requires the information about the spectra of the noise and the original signal and it works well only if the underlying signal is smooth. Wiener method implements spatial smoothing and its model complexity control correspond to choosing the window size. To overcome the weakness of the Wiener filtering (WF), Donoho and Johnstone proposed the wavelet based de-noising scheme in [12] [13].

\section{NOISE}

Noise in an image is a very common problem. During the process of acquisition, transmission or storage retrieval, an image gets corrupted with different types of noise.

\section{CLASSIFICATION OF NOISE}

Noise may be classified as substitutive noise (impulsive noise: e.g., salt \& pepper noise, random-valued impulse noise, etc.) and additive noise (e.g., additive white Gaussian noise). 


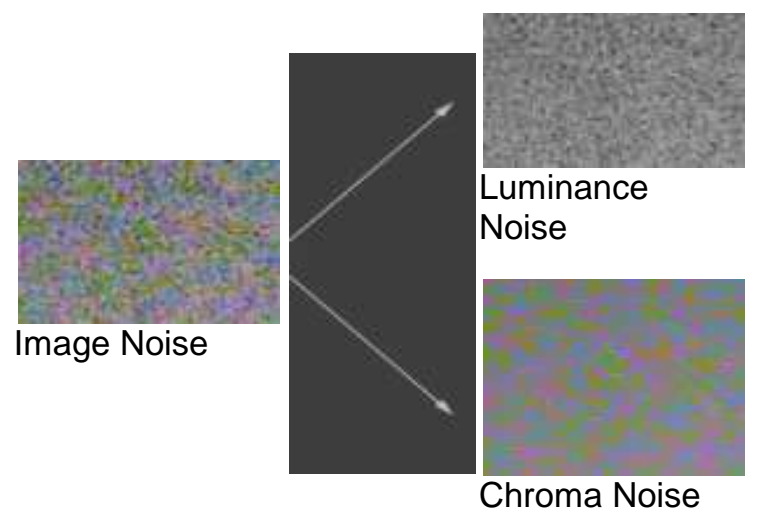

"Figure 3 " "http://www.cambridgeincolour.com/tutorials/image-noise2.html”

\section{EXPERIMENTAL SETUP OF PROPOSED APPROACH}

Our method is explained with the help of a flow chart which is shown below. In this method a gray scale image is taken (all the images are of $256 * 256$ pixel size) which will be shown as original image The step by step blocks of setup are described below with the help of flow.
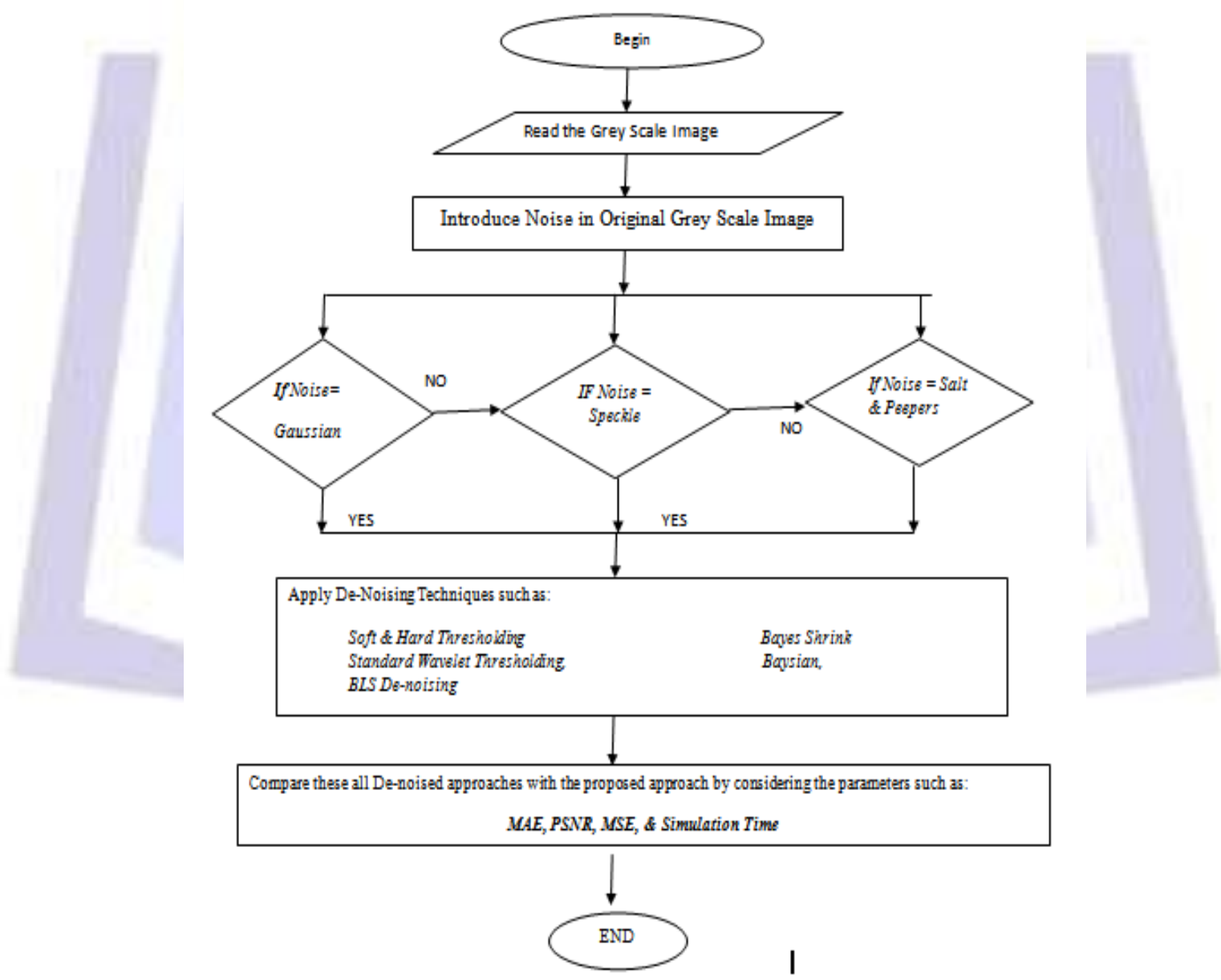

Figure 4 'Flow Chart of Proposed Approach Seup"

\section{DESIGN AND IMPLEMENTATION OF PROPOSED APPROACH}

The above flow chart defines the steps used to run the proposed approach. Now in this section we define the steps o designing and implementing the proposed approach. This proposed approach of de-noising image is implemented in Mat Lab. There are mainly five processes which are as follows:

A image is randomly selected from a data base mainly of 4-5 images having size $256 * 256$. 
Different types of Noises are introduced into the original image.

Various De-noising techniques like Donoho (Hard \& Soft Thresholding), BLS, along with proposed method is applied to the test image .

For the verifications of result a comparative study between various de-noising methods is made which is based upon two performance metrics namely Peak Signal to Noise Ratio (PSNR) and Mean Square error(MSE), Mean Absolute Error(MAE), Simulation Time (Time Complexity).

Reconstruction of wavelet decomposition is done.

MATLAB RESULTS

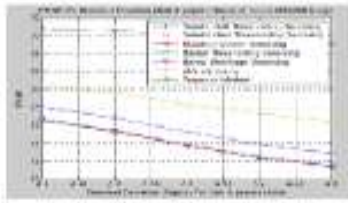

(a)

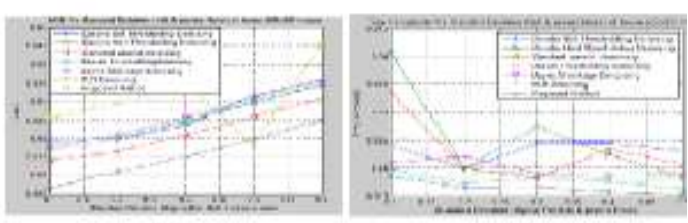

(b)

(6)

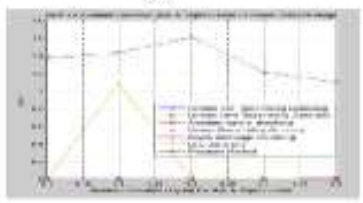

Figure: 5 Results for image "House" Noise type="Salt \& Pepers"

In Fig: 5 the Matlab Results for image "House", Noise type= Salt \& Pepers. (a) "PSNR" is calculated, in (b) "MSE" is calculated in (c) Time Complexity" and in (d) MAE is calculated w.r.t standard deviation of Salt \& Pepers Noise applied to the noiseless image. From above figures, It is very clear that there is the significant improvement in PSNR (a), MSE (b) value, the Time Complexity (c) and the MAE (d) that is obtained with the use of proposed technique over the existing techniques. The MATLAB result shows the comparison of different de-noising methods which define MAE, PSNR \& Time Complexity of Leena by Donoho Soft Thresholding, by Donoho Hard Thresholding, by Wavelet Thresholding, by Bayesian, by Bayes Shrinkage, by BLS-GSM \& by the Proposed Method.

\section{PSNR (in db) Vs Standard deviation( $\sigma)$ Factor for House(256x256) image}

\begin{tabular}{|c|c|c|c|c|c|c|c|c|}
\hline s.xo & $\begin{array}{c}\text { Nolse } \\
\text { Variasece( }(\sigma)\end{array}$ & $\begin{array}{c}\text { Dowoho } \\
\text { Seft }\end{array}$ & $\begin{array}{l}\text { Donoho } \\
\text { llard }\end{array}$ & $\begin{array}{l}\text { Standard } \\
\text { wavelet }\end{array}$ & Hasian & Bayes & BLS & $\begin{array}{l}\text { Proposed } \\
\text { Appreach }\end{array}$ \\
\hline 1 & 0.01 & 78,0048 & 76.569 & 76.5907 & 76.665 & 76.62 & 80.3093 & 86.75 \\
\hline 2 & $0: 02$ & 70.8022 & $73.31 \mathrm{t}$ & 73.3221 & 75.4069 & 75.37 & 79.6252 & 86.5353 \\
\hline 3 & 0.03 & 75,2303 & 73.731 & 73.73 & 73.8329 & 73.788 & 78.3563 & 860343 \\
\hline 4 & 0.04 & 73.8632 & 72391 & 723843 & 72.4757 & 72.443 & 77.3271 & $\$ 55471$ \\
\hline 5 & 0.05 & 728759 & $71+15$ & $71+053$ & 71.9946 & 714896 & 76.4018 & 857007 \\
\hline
\end{tabular}

Table 1 Image Type = 'House', Noise = 'Salt \& Peppers'

MAE (in db) Vs Standard deviation( $\sigma)$ Factor for House (256x256) image:

\begin{tabular}{|c|c|c|c|c|c|c|c|c|}
\hline s.xo & $\begin{array}{c}\text { Noise } \\
\text { Variance(o) }\end{array}$ & $\begin{array}{c}\text { Desabo } \\
\text { Soft }\end{array}$ & $\begin{array}{c}\text { Donobo } \\
\text { Hard }\end{array}$ & $\begin{array}{l}\text { Standaral } \\
\text { wavelet }\end{array}$ & Basian & Baver & BLS & $\begin{array}{l}\text { Proponed } \\
\text { Approach }\end{array}$ \\
\hline 1 & 0.01 & 0.0195 & 0.0171 & 0.0138 & 0.017 & 0.0179 & 0.0256 & 0.0066 \\
\hline 2 & 0.02 & 0.02 & 0.02 & 0.0165 & 0.02 & 0.0209 & 0.0292 & 0.0107 \\
\hline 3 & 0.03 & 0.0255 & 0.0247 & 0.021 & 0.0246 & 0.0256 & 0.0345 & 0.015 \\
\hline 4 & 0.04 & 0.0314 & 0.0297 & 0.0259 & 00296 & 0.0307 & 0.018327 & 0.02 \\
\hline 5 & 0.05 & 0.0359 & 0.0344 & 0.0300 & 0.0343 & 0.0353 & 0.0455 & 0.0249 \\
\hline
\end{tabular}

Table 2 Image Type = 'House', Noise = 'Salt \& Peppers'

Time Complexity (in $\mathrm{db}$ ) Vs Standard deviation( $\sigma)$ Factor for House (256x256) image:

\begin{tabular}{|c|c|c|c|c|c|c|c|c|}
\hline 5.Xo & $\begin{array}{c}\text { Noise } \\
\text { Variance }(\sigma)\end{array}$ & $\begin{array}{l}\text { Donohe } \\
\text { Seft }\end{array}$ & $\begin{array}{c}\text { Domebo } \\
\text { Hard }\end{array}$ & $\begin{array}{l}\text { Standard } \\
\text { wavelet }\end{array}$ & Basian & Bayes & BLS & $\begin{array}{l}\text { Propesed } \\
\text { Approach }\end{array}$ \\
\hline 1 & 0.01 & 0.024201 & 0.040917 & 0.0337 & $0.0198 \mathrm{t}$ & 0.021048 & 0.026891 & 0.018521 \\
\hline 2 & 0.02 & 0,019982 & 0.019952 & 0.019872 & 0.017242 & 0,022273 & 0,01947 & 0.016447 \\
\hline 3 & 0,03 & 0.024503 & 0.027454 & 0.018182 & 0.018182 & 0.018877 & 0.019174 & 0.016523 \\
\hline 4 & 0.04 & 0.024594 & 0.022492 & 0.0234 & 0.01755 & 0.018829 & 0.018327 & 0.015032 \\
\hline 5 & 0.05 & 0.023108 & 0.018229 & 0.020710 & 0.016200 & 0.018908 & 0.017824 & 0.015153 \\
\hline
\end{tabular}


Table 3 Image Type = 'House', Noise = 'Salt \& Peppers'

\section{MSE (in db) Vs Standard deviation( $\sigma)$ Factor for House (256x256) image}

\begin{tabular}{|c|c|c|c|c|c|c|c|c|}
\hline S.No & $\begin{array}{c}\text { Noise } \\
\text { Variance( } \sigma)\end{array}$ & $\begin{array}{c}\text { Donoho } \\
\text { Soft }\end{array}$ & $\begin{array}{c}\text { Donoho } \\
\text { Hard }\end{array}$ & $\begin{array}{c}\text { Standard } \\
\text { wavelet }\end{array}$ & Basian & Bayes & BLs & $\begin{array}{c}\text { Proposed } \\
\text { Approach }\end{array}$ \\
\hline $\mathbf{1}$ & 0.01 & 0.001 & 0.0014 & 0.0014 & 0.0014 & 0.0014 & 0.0016 & 1.3743 \\
\hline $\mathbf{2}$ & 0.02 & 0.0014 & 0.0019 & 0.0019 & 0.0019 & 0.0019 & 1.0887 & 1.4373 \\
\hline $\mathbf{3}$ & 0.03 & 0.002 & 0.0028 & 0.0028 & 0.0027 & 0.0027 & 0.0102 & 1.613 \\
\hline $\mathbf{4}$ & 0.04 & 0.0027 & 0.0038 & 0.0038 & 0.0037 & 0.0037 & 0.0012 & 1.112 \\
\hline $\mathbf{s}$ & 0.05 & 0.0034 & 0.0047 & 0.0047 & 0.0046 & 0.0046 & 0.0015 & 1.0101 \\
\hline
\end{tabular}

Table 4 Image Type = 'House', Noise = 'Salt \& Peppers'

\section{RESULT DISCUSSIONS \& FUTURE SCOPE}

The proposed approach is tested on various standard images (namely Lena, Peppers, House, and Cameraman) but we discussed only on House image in this paper. From the experimental and mathematical results it can be concluded that the proposed approach gives the optimal results as compared to the existing methods. The proposed Non Linear approach produces the maximum PSNR for the output image compared to the linear filters considered. It is also very cleared that the proposed approach have less time complexity. Because the existing filters have more time complexity. So we prove to be better than the Existing methods having less time complexity. Here in Non Linear approach, the sharpness of the image is retained like linear filtering. Proposed method produces the best PSNR compared to BayesShrink. However, the output from BayesShrink method is much closer to the high quality image and there is no blurring in the output image unlike the other two methods. As future research, we would like to work further on the comparison of the de-noising techniques on color images as well.

\section{REFERENCES}

[1] N. Kingsbury,(1999), Image processing with complex wavelets, Phil. Trans. Royal Society London A, 357, $2543-2560$.

[2] M. A. Sid-Ahmed, 1994, Image Processing, New York: McGraw-Hill.

[3] S.G. Chang, Y. Bin, and M. Vetterli, (2000). Spatially adaptive wavelet thresholding with context modeling for image denoising, IEEE Trans. on Image Proc., 9,1532 -1546.

[4] Javier Portilla and Eero P. Simoncelli, (2000), Image Denoising Via Adjustment of Wavelet Coefficient Magnitude Correlation, Proceedings of the 7th International Conference on Image Processing, Vancouver, BC, Canada. 10-13.

[5] J. Astola, P. Kuosmanen, (1997), Fundamentals of Nonlinear Digital Filtering. Boca Raton, FL: CRC Press

[6] S.G. Chang, Y. Bin, and M. Vetterli,(2000) "Adaptive wavelet thresholding for image de-noising and compression," IEEE Trans. on Image Proc., 9, 1522 -1531.

[7] J.L.Starck, E.J.Candes, and D.L.Donoho, (2002) "The curvelet transform for image de-noising," IEEE Trans. on Image Proc., 11-6, $670-684$.

[8] A.Pizurica, W.Philips, I.Lemahieu, and M.Acheroy,(2002). "A joint inter- and intrascale statistical model for bayesian wavelet based image de-noising," IEEE Trans. on Image Proc., vol. 11, pp. 724-735.

[9] M.N.Do and M.Vetterli,(2002). Contourlets: a directional multiresolution image representation," in Proc. Intl. Conf. Image Processing,2002, USA,pp. 22-25.

[10] M.N.Do and M.Vetterli,(2003) "The finite ridgelet transform for image representation," IEEE Trans. on Image Proc., vol. 12, no. 1 , pp. 16-28.

[11] J.Portilla, V.Strela, M.Wainwright, and E.Simoncelli, (2003) "Image denoising using scale mixtures of gaussians in the wavelet domain," IEEE Trans. Image Proc., vol. 12, no. 11, pp. 1338-1351.

[12] G.Fan and X.Xia, (2003). "Wavelet-based texture analysis and synthesis using hidden markov models," IEEE Trans. Circuits and Systems I: Fundamental theory and applications, vol. 50, no. 1, 106-120.

[13] David L. Donoho, Iain M. Johnstone,(1994) “Ideal spatial adaption via wavelet shrinkage”, Biometrika, vol.81, pp 425-455,

[14] David L. Donoho, Iain M. Johnstone,(2001)(1995).“Adapting to unknown smoothness via wavelet shrinkage”, Journal of the American Statistical Association, vol.90, no432, pp.1200-1224, December 1995. National Laboratory.

[15] V. Strela, Birkhäuser Verlag, (2000). "De-noising via block Wiener filtering in wavelet domain” in 3rd European Congress of Mathematics, Barcelona.

[16] I. Daubechies_ and G. Teschke,(2005). Variational image restoration by means of wavelets: simultaneous decomposition, deblurring and de-noising", Elsevier ,Volume 19, Issue 1, 1-16.

[17] A. Buades, B. Coll, and J. Morel,(2006). "Neighborhood Filters and PDE’s,” Numerische Mathematik, 105, No. 1,1-34. 
[18] M. I. H. Bhuiyan, M. Omair Ahmad, and M. N. S. Swamy,(2007). "Wavelet-Based Despeckling Of Medical Ultrasound Images With The Symmetric Normal Inverse Gaussian Prior” IEEE ICASSP, pp I-721-724.

[19] Hossein Rabbani, Mansur Vafadust, Ivan Selesnick and Saeed Gazor,(2007). "Image Denoising Employing A Mixture Of Circular Symmetric Laplacian Models With Local Parameters In Complex Wavelet Domain” IEEE, ICASSP 2007, pp I-805-808.

[20] Santa Agreste, Guido Andaloro, Daniela Prestipino, Luigia Puccio,(2007). "An image adaptive, wavelet-based watermarking of digital images", Elsevier Journal of Computational and Applied Mathematics 210, 13 - 21 